\title{
Low-temperature magnetization of (Ga,Mn)As semiconductors
}

\author{
T. Jungwirth, ${ }^{1,2}$ J. Mašek, ${ }^{3}$ K. Y. Wang, ${ }^{2}$ K. W. Edmonds, ${ }^{2}$ M. Sawicki, ${ }^{4}$ M. Polini, ${ }^{5}$ Jairo Sinova, ${ }^{6}$ A. H. MacDonald, \\ R. P. Campion, ${ }^{2,8}$ L. X. Zhao, ${ }^{2,8}$ N. R. S. Farley, ${ }^{2,8}$ T. K. Johal, ${ }^{8}$ G. van der Laan, ${ }^{8}$ C. T. Foxon, ${ }^{2}$ and B. L. Gallagher ${ }^{2}$ \\ ${ }^{1}$ Institute of Physics ASCR, Cukrovarnická 10, 16253 Praha 6, Czech Republic \\ ${ }^{2}$ School of Physics and Astronomy, University of Nottingham, Nottingham NG7 2RD, United Kingdom \\ ${ }^{3}$ Institute of Physics ASCR, Na Slovance 2, 18221 Praha 8, Czech Republic \\ ${ }^{4}$ Institute of Physics, Polish Academy of Sciences, 02668 Warszawa, Poland \\ ${ }^{5}$ NEST-CNR-INFM and Scuola Normale Superiore, I-56126 Pisa, Italy \\ ${ }^{6}$ Department of Physics, Texas A\&M University, College Station, Texas 77843-4242, USA \\ ${ }^{7}$ Department of Physics, University of Texas at Austin, Austin, Texas 78712-1081, USA \\ ${ }^{8}$ CCLRC Daresbury Laboratory, Warrington WA4 4AD, United Kingdom
}

(Received 10 August 2005; revised manuscript received 20 January 2006; published 12 April 2006)

\begin{abstract}
We report on a comprehensive study of the ferromagnetic moment per Mn atom in (Ga,Mn)As ferromagnetic semiconductors. Theoretical discussion is based on microscopic calculations and on an effective model of Mn local moments antiferromagnetically coupled to valence band hole spins. The validity of the effective model over the range of doping studied is assessed by comparing with microscopic tight-binding/coherent-potential approximation calculations. Using the virtual crystal $\mathbf{k} \cdot \mathbf{p}$ model for hole states, we evaluate the zerotemperature mean-field contributions to the magnetization from the hole kinetic and exchange energies, and magnetization suppression due to quantum fluctuations of Mn moment orientations around their mean-field ground state values. Experimental low-temperature ferromagnetic moments per Mn are obtained by superconducting quantum interference device and x-ray magnetic circular dichroism measurements in a series of $(\mathrm{Ga}, \mathrm{Mn})$ As semiconductors with nominal Mn doping ranging from $\sim 2$ to $8 \%$. Hall measurements in as-grown and annealed samples are used to estimate the number of uncompensated substitutional Mn moments. Based on our comparison between experiment and theory we conclude that all these Mn moments in high quality (Ga,Mn)As materials have nearly parallel ground state alignment.
\end{abstract}

DOI: 10.1103/PhysRevB.73.165205

PACS number(s): 75.50.Pp, 75.30.Gw, 73.61.Ey

\section{INTRODUCTION}

Early experimental studies of $(\mathrm{Ga}, \mathrm{Mn})$ As ferromagnetic semiconductors, reporting large apparent magnetization deficits, ${ }^{1,2}$ motivated a theoretical search for possible intrinsic origins of frustrating magnetic interactions in this material. Using a wide spectrum of computational techniques, ranging from $a b$ initio LDA methods ${ }^{3,4}$ and microscopic tight-binding approximations ${ }^{5}$ to semiphenomenological, $\mathbf{k} \cdot \mathbf{p}$ kinetic-exchange models, ${ }^{6-11}$ the theoretical studies have identified several mechanisms that can lead to noncollinear ground states.

In the Kondo lattice model, which for $(\mathrm{Ga}, \mathrm{Mn})$ As diluted magnetic semiconductors (DMSs) is equivalent to using the disorder-free virtual crystal approximation, the RudermanKittel-Kazuya-Yoshida (RKKY) indirect coupling between Mn $d$-shell moments mediated by itinerant carriers tend to lead to ferromagnetism only when the number of carriers per moment is small, i.e., only when at least the near-neighbor RKKY interaction is ferromagnetic. ${ }^{12}$ One of the surprising features of $(\mathrm{Ga}, \mathrm{Mn})$ As is the property that robust ferromagnetism still occurs when the number of itinerant holes per moment is $\sim 1 .{ }^{13}$ This property follows from the nonparabolic and anisotropic dispersions of the heavy- and lighthole bands and from the strong spin-orbit coupling. $6,9,13$ The complex band-structure leads to a suppression of the $2 k_{F}$ anomaly in the wave vector dependent susceptibility of the hole system which is responsible for the strong RKKY oscillation ${ }^{14}$ of the indirect exchange interaction in simple parabolic band models. Nevertheless, frustrating antiferromagnetic RKKY interactions that promote noncollinear magnetic states ${ }^{15}$ might eventually become important in (Ga,Mn)As systems with very large carrier densities.

The randomness in the distribution of $\mathrm{Mn}$ moments can also result in an instability of the collinear ferromagnetic state. The observation is based in part on the analysis of long wavelength spin-waves with negative energies which frequently occur within the parabolic-band kinetic-exchange model. ${ }^{7}$ Frustration can be further enhanced when positional disorder is combined with anisotropies in $\mathrm{Mn}-\mathrm{Mn}$ interactions. The $p d$ character of electronic states forming the magnetic moment leads to magnetic interaction anisotropies with respect to the crystallographic orientation of the vector connecting two Mn moments. ${ }^{3-5,9}$ When spin-orbit coupling is taken into account, ${ }^{5,8,10,11}$ magnetic interactions also become anisotropic with respect to the relative orientation of the Mn-Mn connecting vector and the magnetic moment. Some degree of noncollinearity is inevitable as a combined consequence of positional disorder and spin-orbit coupling. Nevertheless a large suppression of the ferromagnetic moment is not expected theoretically ${ }^{5}$ in metallic $(\mathrm{Ga}, \mathrm{Mn}) \mathrm{As}$ samples with $\mathrm{Mn}$ concentrations above $1 \%$. The minor role of noncollinearity is due largely to the long-range character of magnetic interactions, which tends to average out the frustrating effect of anisotropic coupling between randomly distributed Mn impurities. ${ }^{5,15}$

In this paper we present detailed calculations of zero temperature magnetization in $(\mathrm{Ga}, \mathrm{Mn}) \mathrm{As}$ ferromagnets and com- 
pare the results with superconducting quantum interference device (SQUID) and $\mathrm{x}$-ray magnetic circular dichroism (XMCD) measurements in a series of samples with nominal Mn doping ranging from $\sim 2-8 \%$. Because of the complexity of the system and experimental error bars we do not attempt to make an accurate quantitative comparison. Instead, our aim is to determine whether substantial noncollinerarity is present or not in the high quality $(\mathrm{Ga}, \mathrm{Mn})$ As ferromagnet materials. Our strategy for the calculations is to neglect effects that would lead to noncollinearity, appealing to expectations that these effects are small, and to account for the key terms that contribute to magnetization in the collinear state. The assumption is consistent with experimental observations of larger ferromagnetic moments in recently synthesized high-quality samples. ${ }^{13,16}$ The substantial magnetization suppression seen in many early $(\mathrm{Ga}, \mathrm{Mn})$ As samples is attributed here primarily to the role played in those samples by interstitial Mn atoms. The consistency of the theoretical and experimental data that we are able to achieve, allows us to rule out any marked magnetic frustrations in the ground state of high-quality $(\mathrm{Ga}, \mathrm{Mn})$ As ferromagnets, and helps to clarify the character of magnetic interactions in this material.

Two distinct theoretical approaches are used in the paper to discuss magnetization in $(\mathrm{Ga}, \mathrm{Mn}) \mathrm{As}$ semiconductors. In the more microscopic approach we account explicitly for the five $d$ orbital electrons on a substitutional $\mathrm{Mn}_{\mathrm{Ga}}$ impurity, and for the strong on-site Coulomb correlations that suppress spin and charge fluctuations of the $L=0, S=5 / 2$ state of the atomic Mn $d$ shell. Magnetism in the mixed crystal arises in this picture from electron hopping between the Mn $d$ states and $p$ orbitals concentrated on the As sublattice that form the top of the host semiconductor valence band. For weak $p$ - $d$ hybridization, a second approach is possible. The Schrieffer-Wolff transformation ${ }^{17}$ allows us to map the microscopic Hamiltonian onto an effective Hamiltonian for local $S=5 / 2$ moments and valence band states whose coupling is described by the kinetic-exchange term $J_{p d} \Sigma_{i, I} \hat{\mathbf{S}}_{I} \cdot \hat{\mathbf{s}}_{i}(\mathbf{r}) \delta\left(\mathbf{r}_{i}-\mathbf{R}_{I}\right)$, where $\hat{\mathbf{S}}_{I}$ and $\hat{\mathbf{S}}_{i}$ are the local moment and valence band state spin operators, respectively. This approach will fail if the $p-d$ hybridization is too strong, but appears to be reliable for $(\mathrm{Ga}, \mathrm{Mn}) \mathrm{As}^{13}$

The paper is organized as follows. In Sec. II we identify the key physical considerations related to ground-state magnetization of $(\mathrm{Ga}, \mathrm{Mn})$ As ferromagnets by focusing first on a single $\operatorname{Mn}\left(d^{5}+\right.$ hole $)$ complex and approximating the total magnetization in the collinear state by a simple sum of individual (identical) $\mathrm{Mn}\left(d^{5}+\right.$ hole $)$ complex contributions. This crude model is used only to qualitatively clarify the connection between $p$ - $d$ hybridization and antiferromagnetic kinetic-exchange coupling, the sign of the hole contribution to total moment per Mn, and the expected mean-field contribution to magnetization per $\mathrm{Mn}$ from the $\mathrm{Mn}$ local moments and from the antiferromagnetically coupled holes. We also explain in this section that quantum fluctuations around the mean-field ground state are generically present because of antiferromagnetic character of the $p$ - $d$ kinetic exchange interaction.

Magnetization calculations for the many-Mn-impurity system are discussed in Sec. III. The relevant considerations here parallel those that apply for isolated $\operatorname{Mn}\left(d^{5}+\right.$ hole $)$ complexes, but differ in detail because of interactions between moments and because of the band character of the holes whose density is, in general, different from the density of $\mathrm{Mn}$ local moments due to the presence of charge compensating defects. Zero temperature ferromagnetic moments per Mn are first studied within the tight-binding-coherent-potential approximation (TBA/CPA) model. ${ }^{18-24}$ This section represents, primarely, an attempt to clarify the differences between the languages that are used to describe the complementary role of local and itinerant moments within the microscopic theory and the effective kinetic-exchange model. The results of the TBA/CPA calculations also indicate that $(\mathrm{Ga}, \mathrm{Mn}) \mathrm{As}$ is in a weak $p$ - $d$ hybridization regime over the whole range of Mn concentrations that we study which helps to establish theoretically the validity of the effective kinetic-exchange model. The virtual crystal approximation and the $\mathbf{k} \cdot \mathbf{p}$ effective Hamiltonian ${ }^{25,26}$ are then used to evaluate contributions to the mean-field magnetization from hole kinetic and exchange energies on a more quantitative level. Note that a significant contribution to magnetization from the itinerant holes can be expected based, e.g., on the reported large spin polarization of the hole currents measured across a (Ga,Mn)As DMS-Ga superconductor interface. ${ }^{27} \mathrm{Fi}$ nally, the model is used to confirm the expected weak role of quantum fluctuations around the mean-field many-body ground state.

Experimental SQUID and XMCD data are presented in Sec. IV. Partial concentrations of substitutional and interstitial Mn impurities and the corresponding number of uncompensated local moments are derived from the nominal $\mathrm{Mn}$ doping and from Hall measurements of the hole density in as-grown and annealed samples. ${ }^{13}$ The collinearity of the ferromagnetic ground state in the $(\mathrm{Ga}, \mathrm{Mn}) \mathrm{As}$ materials we study is tested by comparing experimental data with theoretical calculations. Section V briefly summarizes main conclusions of the paper.

\section{MAGNETIZATION OF AN ISOLATED $\operatorname{Mn}\left(d^{5}+\right.$ HOLE $)$ COMPLEX}

\section{A. $d^{5}+$ hole picture}

The top of the GaAs valence band is dominated by $4 p$ levels which are more heavily weighted on As than on Ga sites. Direct exchange between the holes near the top of the band and the localized $\mathrm{Mn} d$ electrons is weak since $\mathrm{Mn}_{\mathrm{Ga}}$ and As belong to different sublattices. This fact allows $p-d$ hybridization to dominate, explaining the antiferromagnetic sign of this interaction ${ }^{28}$ seen in experiment. ${ }^{29}$

There is a simple physical picture of the $p$ - $d$ exchange interaction which applies when interactions are treated in a mean-field way. Given that the filled, say spin-down, Mn $d$-shell level is deep in the valence band and that the empty spin-up $d$-level is above the Fermi level and high in the conduction band, hybridization (level repulsion of like-spin states) pushes the energy of spin-down valence band states up relative to the energy of spin-up valence band states. The resulting antiferromagnetic coupling between valence band states and local Mn spins is illustrated schematically in Fig. 


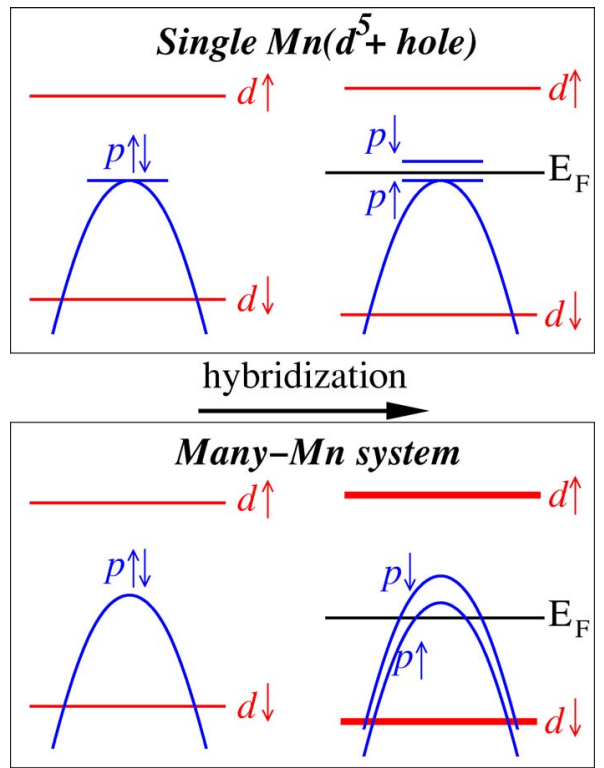

FIG. 1. (Color online) Electron-picture cartoon: splitting of the isolated Mn acceptor level (top panel) and of the top of the valenceband in the many-Mn system (bottom panel) due to $p-d$ hybridization.

1. The same basic picture applies for itinerant valence band states in a heavily-doped metallic DMS, for the impurity band at lower doping and for the acceptor state of an isolated $\mathrm{Mn}_{\mathrm{Ga}}$ impurity.

We note here that the crossover from impurity-band mediated to Bloch valence-band mediated interactions between Mn moments is a gradual one. In the middle of the crossover regime, it is not obvious which picture to use for a qualitative analysis and quantitative calculations are not possible within either picture. Strongly localized impurity-band states away from Fermi energy may play a role in spectroscopic properties, ${ }^{30}$ even when they play a weaker role in magnetic and transport properties. The crossover is controlled not only by the Mn density but (because of the importance of Coulomb interaction screening) also by the carrier density. There is a stark distinction between the compensation dependence predicted by impurity-band and Bloch valence-band pictures. When the impurity-band picture applies, ferromagnetism does not occur in the absence of compensation, ${ }^{31-33}$ because the impurity band is filled. Given this, we can conclude from experiment that the impurity band picture does not apply to high quality (weakly-compensated) (Ga,Mn)As materials which exhibit robust ferromagnetism.

The cartoon band structure in Fig. 1 is plotted in the electron picture while the DMS literature usually refers to the antiferromagnetic $p$ - $d$ coupling between holes and local Mn moments. To avoid confusion that may result from using the hole picture to describe magnetization of carriers in $p$-type (Ga,Mn)As materials, we make a digression here and explain the relation between magnetizations as evaluated using the physically direct electron-picture and magnetizations evaluated using the indirect but computationally more convenient hole-picture. Magnetization at $T=0$ is defined thermodynamically by the dependence of the ground-state energy $E$ on external magnetic field $B$ :

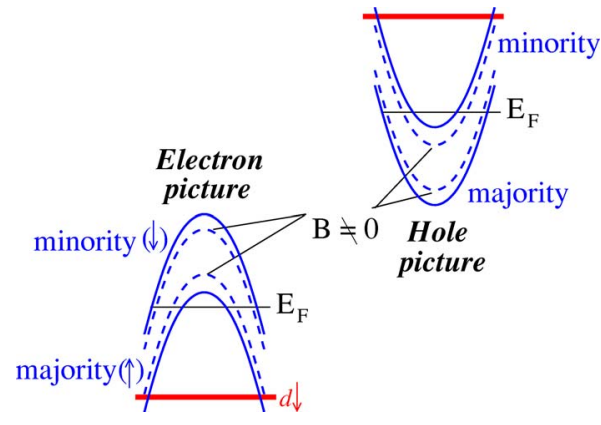

FIG. 2. (Color online) Cartoon of Zeeman coupling of an external magnetic field assuming $g>0$ in the electron and hole pictures for our valence band coupled to Mn moments system. Majority band in both electron and hole pictures moves up in energy resulting in a negative band contribution to the magnetization.

$$
m=-\left.\frac{\partial E}{\partial B}\right|_{B=0} .
$$

In this paper we always assume $\mathbf{B} \|+\hat{\mathbf{z}}$. In mean-field theory the magnetization is related to the change of single-particle energy with field, summed over all occupied orbitals. Orbitals that decrease in energy with field make a positive contribution to the magnetization. For $\mathbf{B} \|+\hat{\mathbf{z}}$, the $d$-electron spins are aligned along $(-z)$ direction (down-spins) and the majority spin band electrons have spin-up due to antiferromagnetic $p-d$ exchange coupling. Then, if the majority spin band moves up in energy with $B$ and the minority band moves down, as illustrated in the left part of Fig. 2, the band kinetic energy increases with $B$ and, according to Eq. (1), the corresponding contribution to the magnetization is negative. In the hole picture, we obtain the same respective sense of shifts of the majority hole and minority hole bands, as illustrated in the right part of Fig. 2, and therefore the correct (negative in our case) sign of the magnetization. The cartoon shows that in order to circumvent the rather confusing notion of a spin of a hole in magnetization calculations, the full Hamiltonian $\hat{H}(B)$ should be derived in the physically direct picture of electron states, where the sign of the coupling of the electron spin to $B$ and the exchange energy are unambiguously defined. The electron picture $\rightarrow$ hole picture transformation $[\hat{H}(B) \rightarrow-\hat{H}(B)]$ and the clearly defined notion of majority and minority bands in either picture guarantees the consistency in sign of the calculated magnetization. Note that the language used here neglects spin-orbit interactions which lead to single-particle orbitals that do not have definite spin character. Although spin-orbit interactions are important they can be neglected in most qualitative considerations, similar to the ones we explain here. In this paper we occasionally make statements which neglect spin-orbit interactions, and they should always be understood in this spirit.

The electron-electron exchange energy has a negative sign and its magnitude increases monotonically when moving from the paramagnetic to the half-metallic (empty minority band) state. This together with Eq. (1) implies that the magnetization contribution from the electron-electron exchange energy has the same sign as the contribution from the kinetic 
energy. Using the same arguments as above we see that in the electron-electron exchange energy case the sign of magnetization is also treated consistently by the electron picture $\rightarrow$ hole picture transformation.

\section{B. Mean-field magnetization}

The mean-field ground state wave function of the $\operatorname{Mn}\left(d^{5}\right.$ +hole) complex is $\left|S_{z}=-S\right\rangle\left|j_{z}=+j\right\rangle$ and the magnetization per Mn equals $m_{\mathrm{MF}}=\left(g_{S} S-g_{j} j\right) \mu_{B}$, where $S$ and $j$ are local $d$ electrons and hole moments and $g_{S}$ and $g_{j}$ are the respective Landé $g$ factors. Assuming atomiclike five $d$-electrons with zero total orbital angular momentum, i.e., $g_{S}=2$, and for a spin $j=1 / 2$ hole $\left(g_{j}=2\right)$ we get $m_{\mathrm{MF}}=4 \mu_{B}$. Hole states near the valence band edge have $p$ character, however, so more realistically we should consider $g_{j} j=4 / 3 * 3 / 2=2$ which gives $m_{\mathrm{MF}}=3 \mu_{B}$. We show in the next section that this basic picture of a suppressed $m_{\mathrm{MF}}$ due to holes applies also to highly Mn-doped (Ga,Mn)As materials although the magnitude of the mean-field hole contribution is weaker because of the occupation of both majority and minority hole bands and, partly, because of spin-orbit coupling effects.

\section{Quantum fluctuations contribution to magnetization}

The two-spin, $S$ and $j$ model can also be used to demonstrate the presence of quantum fluctuations around the meanfield ground state, which is a consequence of the antiferromagnetic sign of the $\mathbf{S} \cdot \mathbf{j}$ coupling. We show that for the $\operatorname{Mn}\left(d^{5}+\right.$ hole $)$ complex, quantum fluctuations are expected to weakly suppress the mean-field magnetization $m_{\mathrm{MF}}$. Detailed many-body calculations discussed in the following section confirm that the role of quantum fluctuations is also weak for the many-Mn systems.

In the limit of $B \rightarrow 0$ the two-spin Hamiltonian is given by

$$
\mathcal{H}=J \hat{\mathbf{S}} \cdot \hat{\mathbf{j}}=\frac{J}{2}\left(\hat{S}_{\text {tot }}^{2}-\hat{S}^{2}-\hat{j}^{2}\right),
$$

where $\hat{\mathbf{S}}_{\text {tot }}=\hat{\mathbf{S}}+\hat{\mathbf{j}}$. For comparison we first consider ferromagnetic coupling $J<0$. Since in this case $S_{\mathrm{tot}}=S+j$, the groundstate eigenenergy, $\quad E_{\mathrm{FM}}=-\frac{|J|}{2}[(S+s)(S+j+1)-S(S+1)-j(j$ $+1)]=-|J| S j$, equals the mean-field energy, i.e., the meanfield state is exact. For ferromagnetic $\mathbf{S} \cdot \mathbf{j}$ coupling, the quantum fluctuation contribution to the magnetization is strictly absent in the many-Mn case only when the hole system is half-metallic (i.e., when the minority band is empty). We can see this by introducing spin raising and lowering operators in the Hamiltonian (2),

$$
\mathcal{H}=J\left[\hat{S}_{z} \hat{j}_{z}+\frac{1}{2}\left(\hat{S}^{+} \hat{j}^{-}+\hat{S}^{-} \hat{j}^{+}\right)\right] .
$$

Quantum fluctuations are absent when the transverse spin terms above annihilate the many-particle ground state. When acting on a state with all localized spins and all band spins polarized in the same direction, both transverse terms produce zero. For partially spin-polarized bands, quantum fluctuation corrections, although not strictly zero, are qualitatively smaller than in the antiferromagnetic case.
For antiferromagnetic coupling $(J>0), S_{\text {tot }}=S-j$ and the corresponding ground-state energy $E_{\mathrm{AF}}=\frac{J J}{2}[(S-j)(S-j+1)$ $-S(S+1)-j(j+1)]=-|J|(S j+j)$ is lower than the mean-field energy. The mean-field ground state is not exact here and quantum fluctuation corrections to the magnetization will be non-zero in general. The difference between magnetizations of the exact and mean-field state is obtained from the exact ground-state wave function as

$$
\begin{aligned}
|\psi\rangle & =\left|S_{\mathrm{tot}}=S-j, S_{\mathrm{tot}, z}=-(S-j)\right\rangle=\sqrt{\frac{S}{S+j}} \mid S_{z}=-S, j_{z} \\
& =+j\rangle-\sqrt{\frac{j}{S+j}}\left|S_{z}=-S+1, j_{z}=+j-1\right\rangle,
\end{aligned}
$$

the mean-field wave function $|\psi\rangle_{\mathrm{MF}}=\left|S_{z}=-S, j_{z}=+j\right\rangle$, and from the respective expectation values of the Zeeman Hamiltonian $g_{S} \mu_{B} B \hat{S}_{z}+g_{j} \mu_{B} B \hat{j}_{z}$, and from Eq. (1):

$$
m-m_{\mathrm{MF}} \equiv m_{Q F}=-\mu_{B} \frac{j}{S+j}\left(g_{S}-g_{j}\right) .
$$

When $j=1 / 2$ and $g_{S}=g_{j}=2$ the quantum fluctuation correction to the magnetization vanishes even though the meanfield ground state is not exact. The correction remains relatively weak also in the case of $j=3 / 2$ and $g_{j}=4 / 3$, for which $m_{Q F}=-0.25 \mu_{B}$.

\section{MAGNETIZATION OF MANY-Mn-IMPURITY SYSTEM}

\section{A. Moments per Mn in the microscopic TBA/CPA model}

As for the $\operatorname{Mn}\left(d^{5}+\right.$ hole $)$ complex, the magnetization of coupled Mn moment systems can be decomposed into meanfield contributions from $\mathrm{Mn}$ local moments and valence band holes and a quantum fluctuations correction. At a mean-field level, the TBA description of $(\mathrm{Ga}, \mathrm{Mn})$ As mixed crystals is particularly useful for explaining the complementary role of local and itinerant moments in this $p$-type magnetic semiconductor. The language that is used to describe this interplay can differ depending on whether a microscopic or a kineticexchange model is employed, and this difference can lead to confusion. This section represents an attempt at clarity. At the same time we find that the TBA/CPA results help establish the validity of the antiferromagnetic $p$-d kineticexchange model and of the virtual crystal approximation for describing collinear ground states in highly doped (Ga,Mn)As ferromagnets. ${ }^{13,24}$

The TBA Hamiltonian ${ }^{18,19}$ includes the $8 \times 8 s p^{3}$ term with second-neighbor-interaction integrals describing the GaAs host ${ }^{20}$ and terms describing hybridization with nonmagnetic impurities and $\mathrm{Mn}$, and electron-electron interactions within the Mn $d$ shell. ${ }^{22}$ The parametrization, summarized in Refs. 20 and 22, provides the correct band gap for a pure GaAs crystal and the appropriate exchange splitting of the Mn $d$ states. In the calculations, the hole density is varied independently of Mn doping by adding non-magnetic donors ( $\mathrm{Si}$ or $\mathrm{Se})$ or acceptors $(\mathrm{C}$ or $\mathrm{Be})$. The $d$-electron magnetic moments of all $\mathrm{Mn}$ atoms are aligned along the $+z$ axis. In the CPA, ${ }^{22,24,34}$ disorder effects appear in the finite spectral 


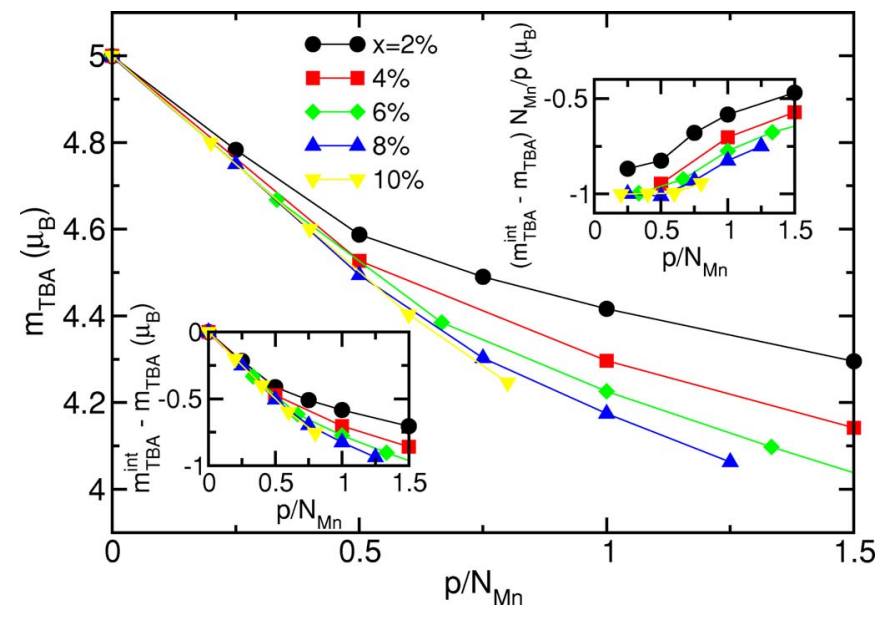

FIG. 3. (Color online) Main panel: mean-field total magnetization per $\mathrm{Mn}$ as a function of hole density relative to the local $\mathrm{Mn}$ moment density. Lower inset: hole contribution to magnetization (see definition in the text) per Mn. Upper inset: hole contribution to magnetization per hole. Results are obtained using the TBA/CPA model.

width of hole quasiparticle states. The TBA/CPA technique can, therefore, capture changes in the $p$ - $d$ interaction with doping due to both chemical alloying effects and positional disorder. In Fig. 3 we show the microscopic TBA/CPA magnetic moments per $\mathrm{Mn}, m_{\mathrm{TBA}}$, in $\mathrm{Ga}_{1-x} \mathrm{Mn}_{x} \mathrm{As}$ ferromagnets plotted as a function of the hole density $p$ relative to the $\mathrm{Mn}$ concentration $N_{\mathrm{Mn}}=4 x / a_{\mathrm{LC}}^{3}\left(a_{\mathrm{LC}}\right.$ is the semiconductor host lattice constant). The $m_{\mathrm{TBA}}$ is obtained here using the electron picture by integrating over occupied states up to the Fermi energy. Spin-orbit coupling is neglected in our TBA Hamiltonian and to simplify the qualitative discussion below only the spin-polarization contribution to magnetization is considered in $m_{\mathrm{TBA}}$.

A common way of microscopically separating contributions from local atomic and itinerant moments is by projecting the occupied electron states onto Mn $d$ orbitals and $s p$ orbitals, respectively. In this decomposition, the resulting local Mn moments are smaller than $5 \mu_{B}$ per Mn due to the admixture of $d$-character in empty states near the valence band edge. The effective kinetic-exchange model employed in the following sections corresponds, however, to a different decomposition of contributions, in effect associating one spectral region with local Mn moments and a different spectral region with itinerant hole moments. The kineticexchange model, in which local moments have $S=5 / 2$, is obtained from the microscopic TBA/CPA model by expressing the total TBA/CPA moment as the difference between a contribution $m_{\mathrm{TBA}}^{\mathrm{int}}$ resulting from integrating over all electronic states up to midgap, i.e., including the entire valence band, and a contribution corresponding to the integral from Fermi energy to midgap. As long as the valence-conduction band gap is nonzero, the former contribution is independent of valence band filling and equals to the moment of an isolated $\mathrm{Mn}$ atom, $5 \mu_{B}$. The latter term, plotted in the lower inset of Fig. 3, represents magnetization of itinerant holes.

The applicability of the effective kinetic-exchange model relies implicitly on the perturbative character of the micro-

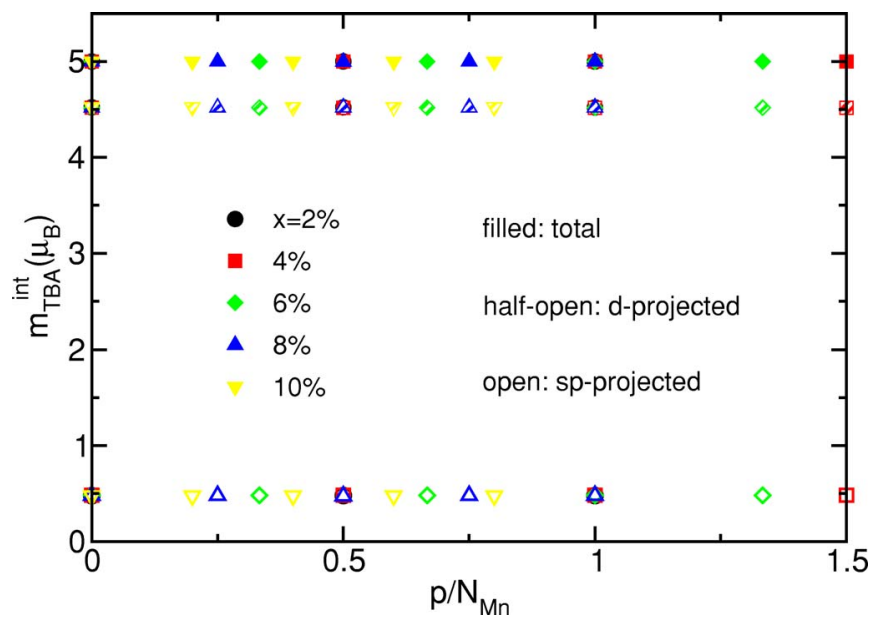

FIG. 4. (Color online) Integrated total and $d$ - and $p d$-projected magnetizations per $\mathrm{Mn}$ as a function of hole density relative to the local Mn moment density. See text for definition of $m_{\mathrm{TBA}}^{\mathrm{int}}$.

scopic $p$ - $d$ hybridization. The level of the $p$ - $d$ hybridization over a typical doping range is illustrated in Fig. 4 where we show the orbital composition of $m_{\mathrm{TBA}}^{\mathrm{int}}$. The filled symbols correspond to including spectral weights from all spd orbitals while the half-open and open symbols are obtained after projecting onto the $d$ and $s p$ orbitals, respectively. If there was no hybridization, $m_{\mathrm{TBA}}^{\text {int }}$ projected on the $d$-orbitals would equal the total $m_{\mathrm{TBA}}^{\mathrm{int}}$ and the $s p$-orbital projected $m_{\mathrm{TBA}}^{\mathrm{int}}$ would vanish. In our TBA/CPA calculations, the $d$-orbital projected $m_{\mathrm{TBA}}^{\text {int }}$ is reduced by only $10 \%$ as compared to the total $m_{\mathrm{TBA}}^{\text {int }}$ and, therefore, the $p$ - $d$ hybridization can be regarded as a weak perturbation. The nearly constant value of the $d$-orbital projected $m_{\mathrm{TBA}}^{\text {int }}$ also suggests that the kinetic-exchange coupling parameter $J_{p d}$ in the effective spin Hamiltonian is nearly independent of doping over the whole range of $\mathrm{Mn}$ and hole densities that we study.

The decrease of $m_{\mathrm{TBA}}$ in Fig. 3 with increasing $p / N_{\mathrm{Mn}}$ clearly demonstrates the antiferromagnetic $p$ - $d$ coupling over the whole range of dopings. The initial common slope for data corresponding to different $\mathrm{Mn}$ concentrations reflects the half-metallic nature of the hole system (only majority hole band occupied) when spin-orbit interactions are neglected. Here the hole contribution to magnetization per volume is proportional to $p$, i.e., magnetization per $\mathrm{Mn}$ is proportional to $p / N_{\mathrm{Mn}}$. The change in slope of $m_{\mathrm{TBA}}$ at larger hole densities, which now becomes Mn-density dependent, reflects population of the minority-spin hole band and, therefore, the additional dependence of hole magnetization on exchange splitting between majority- and minority-hole bands. In this regime the hole magnetization per volume is approximately proportional to $p N_{\mathrm{Mn}}$, i.e., a common trend for different Mn densities is obtained when $m_{\mathrm{TBA}}$ is plotted versus $p$ rather than $p / N_{\mathrm{Mn}}$. Note that the maximum absolute value of the hole contribution to magnetization per hole (see upper inset of Fig. 3) observed in the half-metallic state is $1 \mu_{B}$ in our TBA calculations which assume $j=1 / 2$ and $g_{j}=2$ holes.

Similar conclusions concerning the character of contributions to the magnetization of $(\mathrm{Ga}, \mathrm{Mn}) \mathrm{As}$ have been inferred from $a b$ initio $\mathrm{LDA}+U$ and SIC-LSDA supercell 
calculations. ${ }^{35,36}$ (These microscopic calculations also neglect spin-orbit coupling.) The half-metallic LDA $+U$ band structure in the case of zero charge compensation $\left(p / N_{\mathrm{Mn}}=1\right)$ results in a total magnetization per Mn of $4 \mu_{B},{ }^{35}$ in agreement with the corresponding $m_{\mathrm{TBA}}$ values. In the SIC-LSDA calculations,${ }^{36}$ the system is not completely halfmetallic and, consistently, the total moment per $\mathrm{Mn}$ is larger than $4 \mu_{B}$. The LDA $+U$ and SIC-LSDA local moments on Mn are $4.7 \mu_{B}$ and $4.5 \mu_{B}$, respectively, in good agreement again with the $d$-projected $m_{\mathrm{TBA}}^{\mathrm{int}}$ values. In both $a b$ initio calculations the oppositely aligned moment on the As sublattice extends over the entire supercell, confirming the delocalized character of the holes and the antiferromagnetic sign of the $p$ - $d$ exchange.

Within the TBA/CPA model, $m_{\mathrm{TBA}}$ corresponds to the total magnetization per Mn measured by a SQUID and we therefore expect these experimental values to lie between 4 and $5 \mu_{B}$ and to increase with decreasing number of holes in collinear $(\mathrm{Ga}, \mathrm{Mn})$ As ferromagnets. The XMCD data on the other hand reflect local and $d$-state projected contribution from $\mathrm{Mn}$ and should be compared with the half-open symbols in Fig. 4, showing a negligible dependence on the hole density. These trends are indeed confirmed in our experiments.

Before discussing the experimental data we refine, quantitatively, the above theoretical predictions for the total magnetization. For example, we expect that the number of minority holes at a given total hole density is underestimated in our TBA model. This is caused in part by the quantitative value of the exchange spin-splitting of the valence band in the TBA/CPA calculations which is a factor of 1.5-2 larger than value inferred from experiment. Also since spin-orbit coupling is not included in our TBA model, we obtain three majority bands that are degenerate at the $\Gamma$ point, instead of only two bands (heavy- and light-hole) as in the more realistic spin-orbit coupled band structure. (This deficiency is common to all calculations that neglect spin-orbit coupling.) In addition to having more states available in the majority band which leads to underestimating the minority hole density, the TBA/CPA calculations also omit the reduction of the mean spin-density in the majority band caused by the spinorbit coupling. The total TBA magnetization values would be underestimated due to these effects. On the other hand, assuming only the spin contribution to the hole magnetization leads to an overestimated total magnetization, as discussed in Sec. II. In the following section we attempt to correct for these quantitative shortcomings of the TBA calculations by taking the experimentally measured ${ }^{29,37}$ value of the $p$ - $d$ coupling constant $J_{p d}=54 \pm 9 \mathrm{meV} \mathrm{nm}^{3}$, and by evaluating valence band spin-splitting and spin-orbit coupling effects within the semiphenomenological $\mathbf{k} \cdot \mathbf{p}$ kinetic-exchange model. $^{25,26}$ The weak dependence of the TBA/CPA valence band splitting on positional disorder justifies our use of the virtual crystal approximation in this semiphenomenological modeling of collinear $(\mathrm{Ga}, \mathrm{Mn})$ As ground states.

\section{B. Mean-field magnetization contributions from hole kinetic and exchange energies}

Within the semiphenomenological virtual crystal model the valence band holes experience a mean-field $h_{\mathrm{MF}}$
$=J_{p d} N_{\mathrm{Mn}}\langle S\rangle$, and the band Hamiltonian can then be written as $\hat{H}_{\mathrm{MF}}=\hat{H}_{\mathrm{KL}}(B)+h_{\mathrm{MF}} \hat{s}_{z}$, where $\hat{H}_{\mathrm{KL}}(B)$ is the $B$-dependent sixband Kohn-Luttinger Hamiltonian of the GaAs host band, ${ }^{26,38} \hat{s}_{z}$ is the $z$-component of the hole spin operator, and $\langle S\rangle$ is the mean spin polarization of the local $\mathrm{Mn}$ moments. ${ }^{25,26,39}$ At $T=0,\langle S\rangle=5 / 2$ and the local moment contribution to the magnetization per $\mathrm{Mn}$ is $5 \mu_{B}$. As emphasized above, this finding is not in contradiction with the smaller $d$-electron contribution to the magnetic moment in microscopic calculations.

Because of the spin-orbit interaction, both orbital and Zeeman couplings of the external magnetic field have to be included in $\hat{H}_{\mathrm{KL}}(B)$. The spin-orbit coupling and heavy-holelight-hole mixing at finite wave vectors lead to magnetizations that cannot be expressed using a constant, Mn- and hole-density independent $g$ factor. Instead the kinetic band energy contribution to mean-field magnetization per $\mathrm{Mn}$, $m_{\mathrm{MF}}^{\mathrm{kin}}$, is obtained by numerically integrating over all occupied hole eigenstates of $\hat{H}_{\mathrm{MF}}$ and by finding the coefficient linear in $B$ of this kinetic energy contribution to the total energy. ${ }^{26}$ The $\hat{z}$ axis defining the direction of $B$ (and the magnetization axis) corresponds to the $\langle 001\rangle$ crystal axis in our calculations. We have checked numerically that the effect of typical $\langle 001\rangle$ growth-direction lattice-matching strains on $m_{\mathrm{MF}}^{\mathrm{kin}}$ is less than $1 \%$ and, therefore, the data presented below were calculated assuming full cubic symmetry. Since strain effects dominate the magnetocrystalline anisotropy, ${ }^{25,26}$ the dependence of $m_{\mathrm{MF}}^{\mathrm{kin}}$ on magnetization orientation can be expected to also be small. This is confirmed, within the experimental error bars, by both SQUID and XMCD measurements.

Results for several typical Mn dopings and hole densities are shown in Fig. 5. They agree quantitatively with earlier calculations reported in Ref. 26. As anticipated in Sec. II, $m_{\mathrm{MF}}^{\mathrm{kin}}$ is negative, i.e., it suppresses the total magnetic moment. The magnitude of the hole mean-field magnetization per hole $\left|m_{\mathrm{MF}}^{\mathrm{kin}}\right| N_{\mathrm{Mn}} / p$, is smaller than $2 \mu_{B}$ obtained in Sec. II due to occupation of both majority and minority heavy- and light-hole bands at these typical $(\mathrm{Ga}, \mathrm{Mn})$ As hole densities (see upper inset of Fig. 5). In this case, as also emphasized in Sec. III A, $m_{\mathrm{MF}}^{\mathrm{kin}}$ is expected to fall roughly into a common trend for different Mn densities when plotted against $p$. Data shown in the main panel of Fig. 5 and in the lower inset confirm this expectation and indicate a $\sim 0.2$ to $0.4 \mu_{B}$ suppression of the mean-field moment per Mn due to the hole kinetic energy contribution to magnetization. ${ }^{26}$

To estimate the hole exchange energy contribution to magnetization per $\mathrm{Mn}, m_{\mathrm{MF}}^{\mathrm{ex}}$, we use an expression of the total exchange energy derived in the absence of spin-orbit coupling and assuming spin-up and spin-down heavy-hole bands with effective mass $0.5 m_{e}$,

$$
E_{\mathrm{ex}}=2^{1 / 3} \frac{E_{\mathrm{ex}}^{P}(n)}{p^{4 / 3}}\left[p_{\uparrow}^{4 / 3}(B)+p_{\downarrow}^{4 / 3}(B)\right],
$$

where $p_{\uparrow(\downarrow)}$ is the density of the majority(minority) band, $p_{\uparrow}+p_{\downarrow}=p$, and the exchange energy of the paramagnetic state is given by 


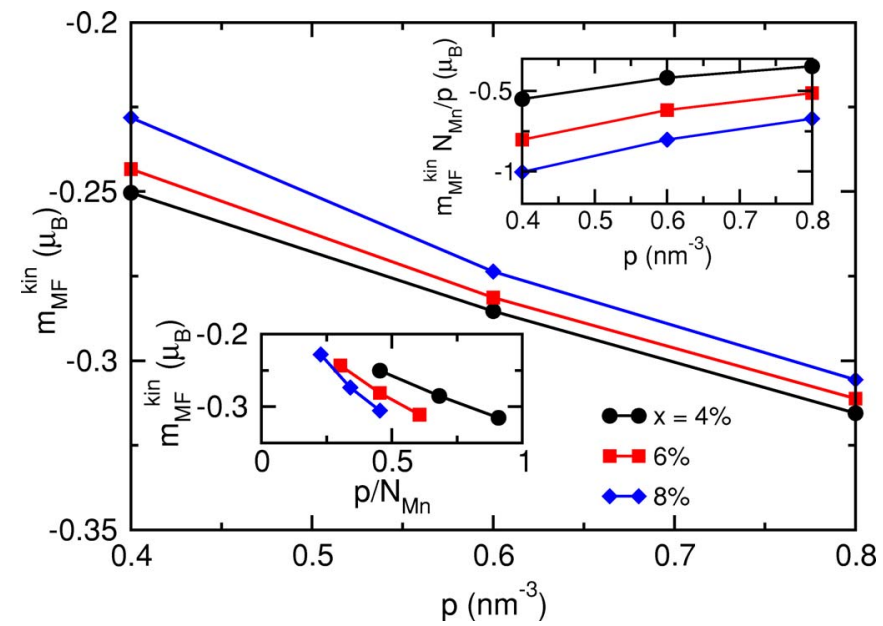

FIG. 5. (Color online) Main panel: mean-field kinetic energy contribution to the hole magnetization per $\mathrm{Mn}$ as a function of hole density. Lower inset: same quantity plotted as a function of hole density relative to the local Mn moment density. Upper inset: meanfield kinetic energy contribution to the hole magnetization per hole as a function of hole density. These results were obtained using the six-band Kohn-Luttinger parameterization of the valence band and the kinetic-exchange model.

$$
E_{\mathrm{ex}}^{P}(n)=-\frac{e^{2}}{4 \pi \varepsilon} \frac{3}{4}\left(\frac{3}{\pi}\right)^{1 / 3} p^{4 / 3} .
$$

The $B$-dependent hole densities can be written as

$$
\begin{aligned}
& p_{\uparrow}(B)=p_{\uparrow}(0)-\left|g_{j} \mu_{B} B j\right| \frac{2 G^{\uparrow} G^{\downarrow}}{G^{\uparrow}+G^{\downarrow}}, \\
& p_{\downarrow}(B)=p_{\downarrow}(0)+\left|g_{j} \mu_{B} B j\right| \frac{2 G^{\uparrow} G^{\downarrow}}{G^{\uparrow}+G^{\downarrow}},
\end{aligned}
$$

where $G^{\uparrow(\downarrow)}$ is the density of states at the Fermi energy of the majority(minority) band. Combining Eqs. (6)-(8) and Eq. (1) we obtain

$$
m_{\mathrm{MF}}^{\mathrm{ex}}=\frac{4}{3} \frac{2^{1 / 3}}{p^{4 / 3}} E_{\mathrm{ex}}^{P}(n) g_{j} \mu_{B} j \frac{2 G^{\uparrow} G^{\downarrow}}{G^{\uparrow}+G^{\downarrow}}\left[p_{\uparrow}(0)^{1 / 3}-p_{\downarrow}(0)^{1 / 3}\right] .
$$

In Fig. 6 we plot $m_{\mathrm{MF}}^{\mathrm{ex}}$ values calculated assuming spin$1 / 2$ holes, i.e., $g_{j} j=1$. This contribution to the total meanfield magnetization is again negative, is nearly independent of $x$ and $p$ within the range of doping considered, and its magnitude is about a factor of 10 smaller than the magnitude of the term originating from the hole kinetic band energy. A more realistic estimate of $m_{\mathrm{MF}}^{\mathrm{ex}}$ can be obtained by using $g_{j} j=g_{\text {fit }}(3 / 2)$ in Eq. (9). Here $g_{\text {fit }}$ follows from fitting the $m_{\mathrm{MF}}^{\text {kin }}$ calculated within the parabolic heavy-hole band model to the full six-band numerical results in Fig. 5. The values of $g_{\text {fit }}$ as a function of $x$ and $p$ are plotted in Fig. 7. These are similar to the $g_{j=3 / 2}=4 / 3$ value that follows from the local atomic model and, therefore, $m_{\mathrm{MF}}^{\mathrm{ex}}$ calculated from Eq. (9) using $g_{j} j=g_{\text {fit }}(3 / 2)$ is approximately a factor of 2 larger than $m_{\mathrm{MF}}^{\mathrm{ex}}$ calculated assuming spin-1/2 holes. Combining all

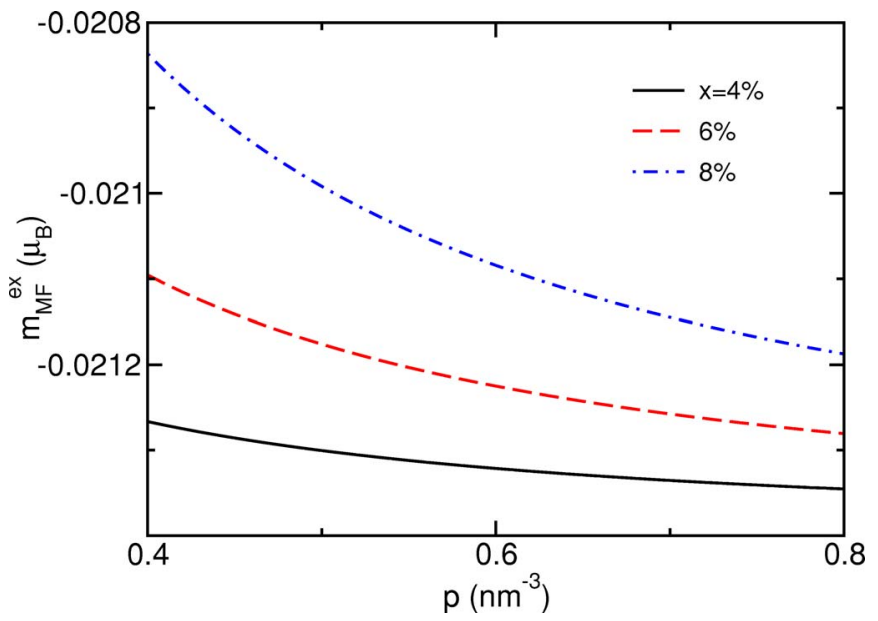

FIG. 6. (Color online) Mean-field hole-hole exchange energy contribution to the hole magnetization per $\mathrm{Mn}$ as a function of hole density. These results were obtained using the spin-1/2, $m^{*}$ $=0.5 m_{e}$ parabolic band kinetic-exchange model.

these considerations we conclude that the zero-temperature magnetization per $\mathrm{Mn}$ in the mean-field kinetic-exchange model has a positive contribution equal to $5 \mu_{B}$ from the Mn local moments and a negative contribution from band holes which suppresses the moment per Mn by $\sim 5-10 \%$.

\section{Quantum fluctuation contribution to the magnetization}

In Sec. II we argued that quantum fluctuation corrections to the isolated $\mathrm{Mn} d^{5}+$ hole complex magnetization should be small. Here we demonstrate that this conclusion also applies to the many-Mn system. In these calculations we use the virtual crystal kinetic-exchange model and assume spin-1/2 heavy holes with no spin-orbit coupling and with the parabolic band dispersion $\left(m^{*}=0.5 m_{e}\right)$. The many-body Hamiltonian of the model reads

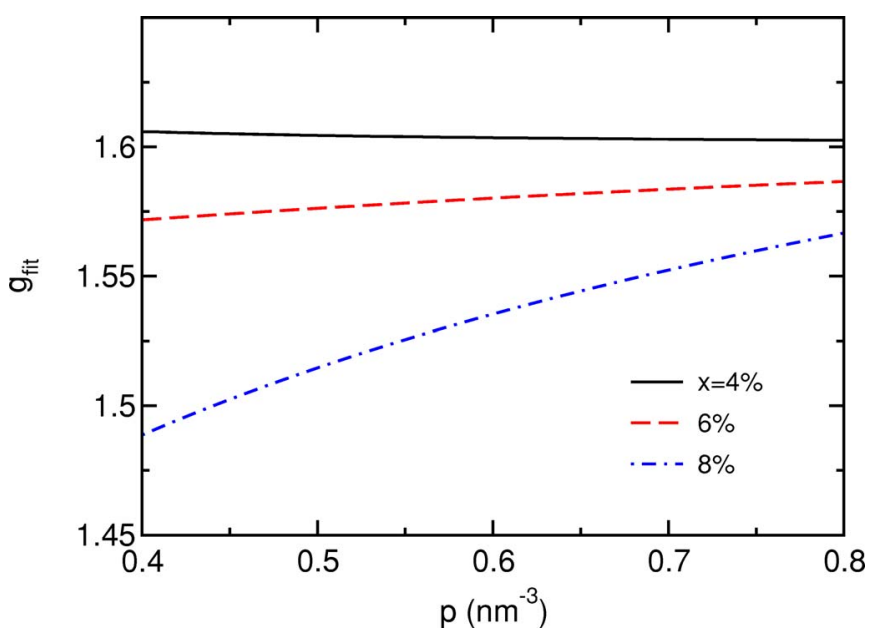

FIG. 7. (Color online) Effective hole Landé $g$ factor obtained by fitting the kinetic energy term in hole magnetization calculated with the parabolic band kinetic-exchange model with $j=3 / 2$ and $m^{*}$ $=0.5 m_{e}$ to the numerical results of the six-band Kohn-Luttinger model from Fig. 5. 


$$
\begin{aligned}
\hat{H}= & \int d^{3} r\left[\sum_{\sigma} \hat{\Psi}_{\sigma}^{\dagger}(\vec{r})\left(-\frac{\hbar^{2} \vec{\nabla}^{2}}{2 m^{*}}-\mu\right) \hat{\Psi}_{\sigma}(\vec{r})+g_{j} \mu_{B} \vec{B} \cdot \vec{j}(\vec{r})\right. \\
& \left.+\left|J_{p d}\right| \sum_{I} \vec{S}\left(\overrightarrow{R_{I}}\right) \cdot \vec{j}(\vec{r}) \delta\left(\vec{r}-\vec{R}_{I}\right)\right]+g_{S} \mu_{B} \sum_{I} \vec{B} \cdot \vec{S}\left(\vec{R}_{I}\right) .
\end{aligned}
$$

The imaginary time path-integral formulation of quantum statistical physics combined with a Holstein-Primakoff bosonic representation for the $\mathrm{Mn}$ local moments allows ${ }^{6}$ us to formally express the free energy of interacting local and itinerant spins in terms of a path integral over coherent state labels $\bar{z}, z$ :

$$
\mathcal{Z}=\int \mathcal{D}(\bar{z} z) \exp \left(-S_{\text {eff }}[\bar{z} z]\right)
$$

The effective action $S_{\text {eff }}$ in Eq. (11) is obtained by integrating out fermionic (hole) degrees of freedom in Eq. (10). In the Gaussian fluctuation approximation ${ }^{6}$

$$
S_{\mathrm{eff}}[\bar{z} z]=\frac{1}{\beta} \sum_{m} \int_{|\mathbf{q}| \leqslant q_{c}} \frac{d^{3} \mathbf{q}}{(2 \pi)^{3}} \bar{z}\left(\mathbf{q}, \nu_{m}\right) D^{-1}\left(\mathbf{q}, i \nu_{m}\right) z\left(\mathbf{q}, \nu_{m}\right) .
$$

Here the inverse of the spin-wave propagator $D\left(\mathbf{q}, \nu_{m}\right)$ is given by

$$
D^{-1}\left(\mathbf{q}, i \nu_{m}\right)=-i \nu_{m}+\varepsilon_{s w}(B)+\Sigma_{s w}\left(\mathbf{q}, i \nu_{m}, B\right),
$$

$q_{c}=\left(6 \pi^{2} N_{\mathrm{Mn}}\right)^{1 / 3}$ is a Debye cutoff which ensures that we include the correct number of local-moment degrees of freedom and

$$
\varepsilon_{s w}(B)=-g_{S} \mu_{B} B+\frac{\left|J_{p d}\right|}{2}\left[p_{\uparrow}(B)-p_{\downarrow}(B)\right]
$$

is the mean-field local moment spin-flip energy. The frequency-dependent self-energy $\Sigma_{s w}(\mathbf{q}, i \Omega, B)$ in Eq. (13) is given by

$$
\Sigma_{s w}(\mathbf{q}, i \Omega, B)=\frac{N_{\mathrm{Mn}} J_{p d}^{2} S}{2} \times \int \frac{d^{3} \mathbf{k}}{(2 \pi)^{3}} \frac{f\left[\varepsilon_{\mathbf{k}}-\mu+\Delta(B) / 2\right]-f\left[\varepsilon_{\mathbf{k}+\mathbf{q}}-\mu-\Delta(B) / 2\right]}{i \Omega+\varepsilon_{\mathbf{k}}-\varepsilon_{\mathbf{k}+\mathbf{q}}+\Delta(B)},
$$

with $\varepsilon_{\mathbf{k}}=\hbar^{2} k^{2} / 2 m^{*}$ and

$$
\Delta(B)=N_{\mathrm{Mn}}\left|J_{p d}\right| S-g_{j} \mu_{B} B .
$$

The translational and rotational invariance of our model implies that $\Sigma_{s w}(\mathbf{q}, i \Omega)$ depends only of $|\mathbf{q}|$. The functional integration in Eq. (11) can be performed exactly using Eq. (12),

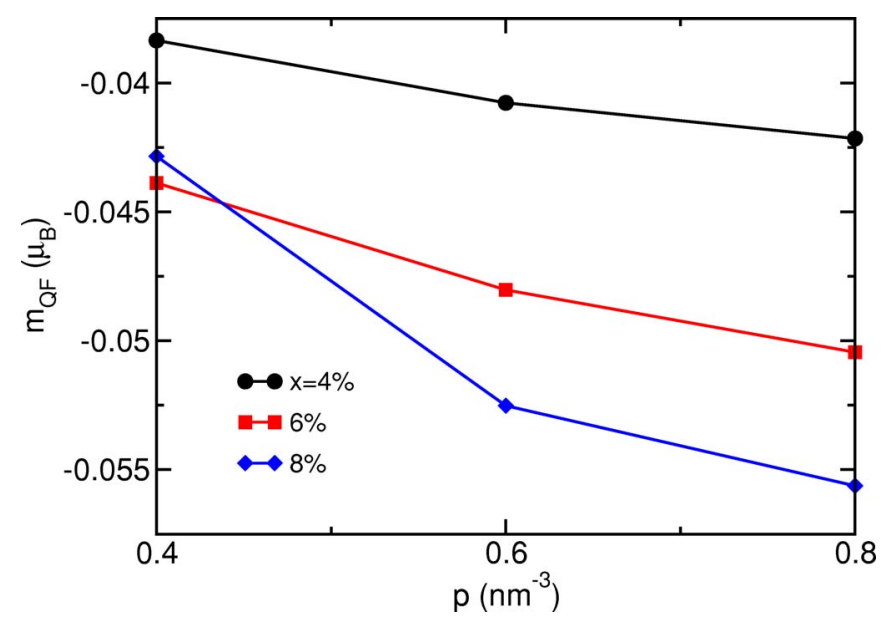

FIG. 8. (Color online) Quantum fluctuation contribution to magnetization per $\mathrm{Mn}$ as a function of hole density. These results were obtained using the spin-1/2, $m^{*}=0.5 m_{e}$ parabolic band kineticexchange model.

$$
\mathcal{Z}=\frac{1}{\beta_{n,|\mathbf{q}| \leqslant q_{c}}} \frac{1}{-i \nu_{n}+\varepsilon_{s w}(B)+\Sigma_{s w}\left(q, i \nu_{n}, B\right)} .
$$

The quantum fluctuation correction to the free energy then reads,

$$
\delta F_{\mathrm{QF}} \equiv F-F_{0}=-\frac{1}{\beta} \ln \frac{\mathcal{Z}}{\mathcal{Z}_{0}},
$$

where $\mathcal{Z}_{0}$ is given by Eq. (16) with the fluctuations term, $\Sigma_{s w}\left(q, i \nu_{n}, B\right)$, set to zero.

In Fig. 8 we plot the quantum fluctuations contribution to the magnetization per Mn obtained from

$$
m_{\mathrm{QF}}=-\left.\frac{\partial \delta E_{\mathrm{QF}}}{\partial B}\right|_{B=0} .
$$

As expected $m_{\mathrm{QF}}$ is small in the many-Mn system and we can conclude that quantum fluctuations lead to a $\sim 1 \%$ suppression of the mean-field moment per Mn.

\section{EXPERIMENTAL DATA}

\section{A. Sample growth and preparation}

In the theoretical sections of this paper we evaluated the zero-temperature magnetization per $\mathrm{Mn}$ in $(\mathrm{Ga}, \mathrm{Mn}) \mathrm{As}$ ferromagnets, using approximation schemes that would fail if the true ground state magnetization was highly noncollinear. We have accounted for the key terms that contribute to the mag- 
netization in the collinear state and found large moments per Mn which are reduced by $\sim 10 \%$ as compared to the moment of atomic Mn. We will now show that these theoretical results are consistent with low temperature magnetometry and XMCD experiments.

A series of $(\mathrm{Ga}, \mathrm{Mn})$ As films with $\mathrm{Mn}$ content varying between $1.7-6.7 \%$ in the SQUID experiments and between 2.2 and $8.4 \%$ in the XMCD experiments were grown by low-temperature molecular beam epitaxy (MBE) using $\mathrm{As}_{2}$. The layer structure of the thin films consists of 25 or $50 \mathrm{~nm}$ $(\mathrm{Ga}, \mathrm{Mn}) \mathrm{As} / 50 \mathrm{~nm}$ low temperature GaAs/100 nm high temperature $\left(580{ }^{\circ} \mathrm{C}\right) \mathrm{GaAs} / \mathrm{SI}-\mathrm{GaAs}(100)$ substrate. The growth temperature of the $(\mathrm{Ga}, \mathrm{Mn}) \mathrm{As}$ layer and the GaAs buffer was $180-300{ }^{\circ} \mathrm{C}$, decreasing with increasing Mn concentration in order to minimize As antisite densities while maintaining two-dimensional growth and preventing phase segregation. Further details on the growth are presented elsewhere. ${ }^{40,41}$

The Mn concentrations were deduced from the in situ measured $\mathrm{Mn} / \mathrm{Ga}$ incident flux ratio, which was calibrated using secondary ion mass spectrometry (SIMS) measurements on $1 \mu \mathrm{m}$ thick $(\mathrm{Ga}, \mathrm{Mn}) \mathrm{As}$ films, grown under otherwise identical conditions to the samples considered here. A detailed comparison of the results of a number of different calibration techniques, presented in detail elsewhere, ${ }^{42}$ allows us to assign an uncertainty of $10 \%$ to the quoted total Mn doping $x$. The SIMS measurements yield no information on the lattice site of the incorporated Mn, and we expect that $\mathrm{Mn}$ will be incorporated either on interstitial $\mathrm{Mn}_{\mathrm{I}}$ or on substitutional $\mathrm{Mn}_{\mathrm{Ga}}$ sites. ${ }^{43}$ Post-growth annealing of the samples is performed in air at $190{ }^{\circ} \mathrm{C}$ for $50-150 \mathrm{~h}$, which is an established procedure for removal of $\mathrm{Mn}_{\mathrm{I}}$ from the $(\mathrm{Ga}, \mathrm{Mn})$ As layer. Curie temperatures in the as-grown materials are within the range of $40-80 \mathrm{~K}$ and in the annealed samples between 40 and $150 \mathrm{~K} .^{13}$

\section{B. Magnetometry}

The magnetic moment of the samples is measured in a SQUID magnetometer, at $5 \mathrm{~K}$ and under a $0.3 \mathrm{~T}$ external magnetic field. The external field is necessary to overcome in-plane anisotropy fields, so that the magnetization is aligned with the measurement axis of the SQUID. The diamagnetic contribution from the substrate is subtracted. Measured magnetic moments normalized to the total Mn concentration as obtained from SIMS calibration, $m_{\text {SQUID }}$, are shown in Fig. 9. For these data, magnetic field and magnetization are aligned along the $\langle 100\rangle$ crystallographic axis which is the easy magnetic axis at low temperatures. Within experimental uncertainty, $m_{\mathrm{SQUID}}$ was found to be independent of the magnetization orientation, in agreement with theoretical expectations. The moment decreases with increasing Mn concentration, and increases on annealing, similar to earlier reports. ${ }^{2}$ This is consistent with the anticipated formation of interstitial $\mathrm{Mn}$ for doping above $\sim 2 \%,{ }^{13}$ given the antiferromagnetic coupling between $\mathrm{Mn}_{\mathrm{I}}$ and $\mathrm{Mn}_{\mathrm{Ga}}{ }^{23}$ and with breaking of this coupling by low-temperature annealing. ${ }^{43,44}$

In order to compare the experimental data with the theoretical results of previous sections we have to replot the mea-

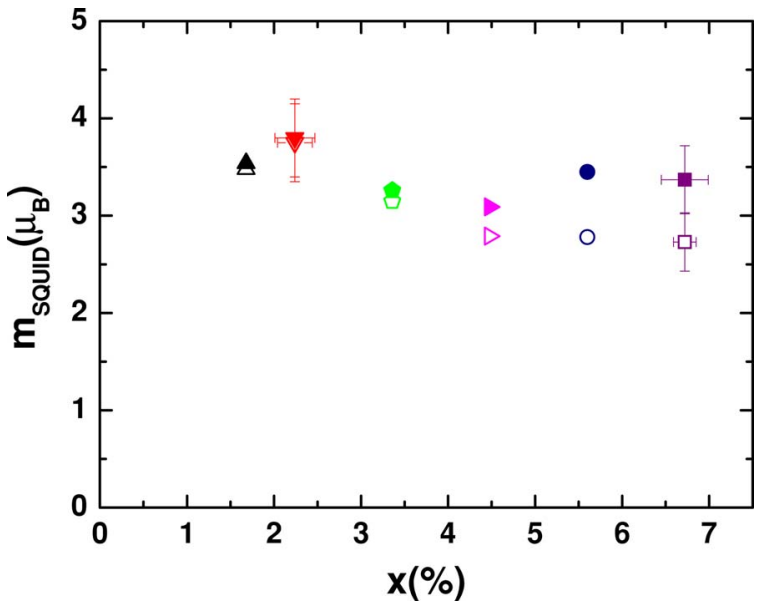

FIG. 9. (Color online) SQUID magnetization per nominal total Mn density in as-grown (open symbols) and annealed (filled symbols) (Ga,Mn)As materials plotted as a function of the nominal Mn doping.

sured magnetizations as a function of the density of uncompensated $\mathrm{Mn}_{\mathrm{Ga}}$ local moments $x_{\text {eff }}$. To do this we need to determine the densities of substitutional $\mathrm{Mn}_{\mathrm{Ga}}$ and interstitial $\mathrm{Mn}_{\mathrm{I}}, x_{s}$, and $x_{i}$, in our $(\mathrm{Ga}, \mathrm{Mn}) \mathrm{As}$ materials. Given these values, we assume ${ }^{13}$ that each $\mathrm{Mn}_{\mathrm{I}}$ present in the system is antiferromagnetically coupled to one $\mathrm{Mn}_{\mathrm{Ga}}$ and that both should be excluded from the active Mn fraction for comparison between theory and experiment, i.e., that $x_{\text {eff }}=x_{s}-x_{i}$.

To obtain the individual Mn impurity concentrations we rely on Hall effect and magnetoresistance measurements at high magnetic field (up to $16.5 \mathrm{~T}$ ) and low temperatures (down to $0.3 \mathrm{~K}$ ), from which we evaluate the experimental hole density $p$, after using a fitting procedure to separate normal and anomalous contributions to the Hall resistance. ${ }^{45}$ We then assume that the single acceptors $\mathrm{Mn}_{\mathrm{Ga}}$ and double donors $\mathrm{Mn}_{\mathrm{I}}$ are the only impurities that contribute to $p$, i.e., $p=\left(4 / a_{l c}^{3}\right)\left(x_{s}-2 x_{i}\right)$. From this expression and from the total Mn concentration obtained by SIMS calibration $\left(x=x_{s}+x_{i}\right)$ we can estimate $x_{\text {eff }}$ for both as-grown and annealed samples. A detailed discussion of the uncertainties associated with this procedure is given elsewhere. ${ }^{13}$ The magnetic moment per effective Mn moment density $m_{\text {SQUID }}^{\text {eff }}$ is shown in Fig. 10 as a function of $p / N_{\mathrm{Mn}}^{\mathrm{eff}}$ where $N_{\mathrm{Mn}}^{\mathrm{eff}}=\left(4 x_{\mathrm{eff}} / a_{l c}^{3}\right)$. In agreement with the predictions of the theory section, $m_{\mathrm{SQUID}}^{\text {eff }}$ falls within the range $4-5 \mu_{B}$ for all samples studied. Furthermore, although there is appreciable scatter, it can be seen that samples with lower hole densities tend to show higher $m_{\text {SQUID }}^{\text {eff }}$, consistent with a negative contribution to magnetization from antiferromagnetically coupled band holes.

\section{X-ray magnetic circular dichroism}

The local and $d$-projected contribution from $\mathrm{Mn}$ to the magnetic moment in $(\mathrm{Ga}, \mathrm{Mn}) \mathrm{As}$ can be probed experimentally by XMCD measurements. For completeness of our analysis of the low temperature magnetization we briefly introduce in this section the XMCD technique and discuss the values of the local Mn moments inferred from these experi- 


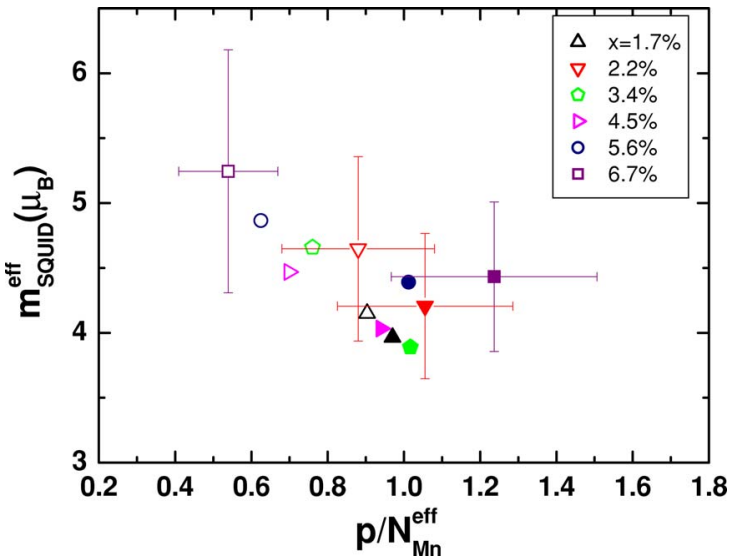

FIG. 10. (Color online) SQUID magnetization per effective density of uncompensated $\mathrm{Mn}_{\mathrm{Ga}}$ local moments in as-grown (open symbols) and annealed (filled symbols) (Ga,Mn)As materials plotted as a function of hole density per effective density of uncompensated $\mathrm{Mn}_{\mathrm{Ga}}$ local moments.

ments. Details of the experimental setup and procedures used to interpret the XMCD data can be found in Ref. 16.

XMCD measurements were performed using $(99 \pm 1) \%$ circular polarized $\mathrm{x}$ rays from beamline ID8 at the European Synchrotron Radiation Facility. The samples were briefly etched in concentrated $\mathrm{HCl}$ prior to the measurements in order to remove Mn oxide rich surface layers, which may obscure the signal from the $(\mathrm{Ga}, \mathrm{Mn})$ As due to the relatively short probing depth of the measurement. ${ }^{46}$ After etching, the quantitative agreement between total electron yield and fluorescent yield measurements indicated a uniform distribution of $\mathrm{Mn} .{ }^{16,46}$

Figure 11 shows $M n L_{3,2}$ x-ray absorption spectra for annealed $(\mathrm{Ga}, \mathrm{Mn})$ As samples with $x=2.2$ and $8.4 \%$, for parallel and antiparallel orientations of the external magnetic field and the x-ray helicity vector. The sample temperature is $6 \mathrm{~K}$, and the external magnetic field is $\pm 1 \mathrm{~T}$, applied perpendicular to the surface of the sample. In agreement with the SQUID measurements and theoretical expectations, the XMCD data are independent, within experimental uncertainty, of the direction of magnetization. ${ }^{47}$ Currently, the values of $x=2.2$ and $8.4 \%$ represent typical low-doped and high-doped $(\mathrm{Ga}, \mathrm{Mn})$ As materials which show robust ferromagnetism and weak compensation after annealing. ${ }^{13}$ The ferromagnetic transition temperature in these annealed highquality materials increases linearly with $x_{\text {eff }}$ showing no signs of saturation, and the materials exhibit no special features at intermediate $x$. Similarly, XMCD data at intermediate $\mathrm{Mn}$ doping ${ }^{47}$ are consistent with the data we show here for $x=2.2$ and $8.4 \%$.

As shown in Fig. 11, a very large change in the absorption is observed on reversing the external field, with an asymmetry (difference to sum ratio) of up to $55 \%$ at the $L_{3}$ peak. $L_{3,2}$ absorption corresponds to transitions from the $2 p$ core states to the unfilled $3 d$ states, so the Mn $L_{32}$ spectra gives direct information on the polarization of the Mn $3 d$ band. Applying the XMCD sum rules to the spectra allows quantitative and separate determination of the Mn $3 d$ ground state orbital and spin magnetic moments $m^{\text {orb }}$ and $m^{\text {spin }} \cdot 16,48$ The moments are

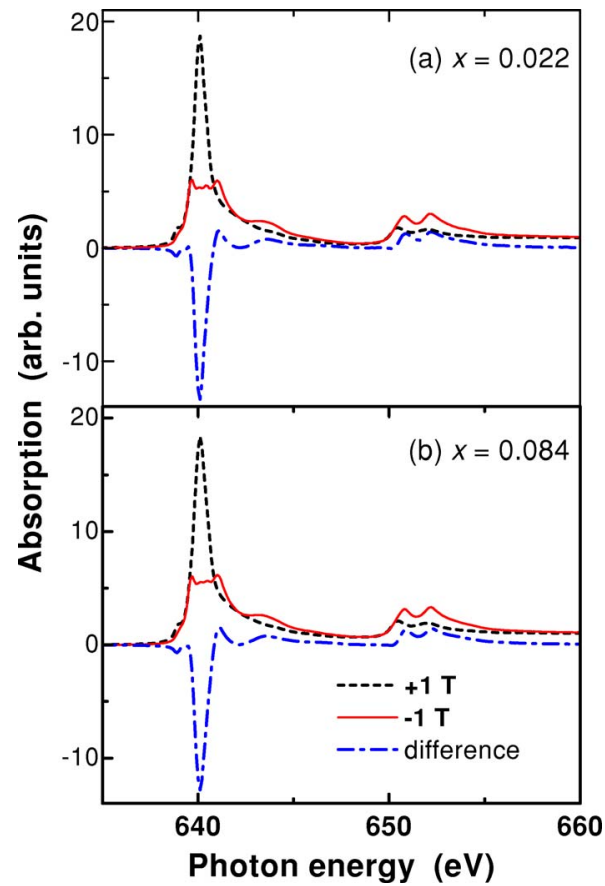

FIG. 11. (Color online) Mn $L_{3,2} \mathrm{x}$-ray absorption spectra for annealed (Ga,Mn)As samples with nominal Mn doping (a) $2.2 \%$ and (b) $8.4 \%$, for parallel and antiparallel orientations of the external magnetic field and the $\mathrm{x}$-ray helicity vector. The dotted-dashed (blue) lines show the difference between parallel and antiparallel spectra.

obtained on a per atom basis, without requiring separate measurement of the Mn concentration, by normalizing to summed absorption signal. The procedure includes, e.g., corrections for the mixing of the $2 p_{3 / 2}$ and $2 p_{1 / 2}$ states which prevents the separate integration over each of the spin-orbit split core levels. The correction factors are obtained based on calculations for an isolated pure $d^{5} \mathrm{Mn}$ state and on corresponding XMCD experiments in, e.g., dilute Mn adsorbates on Fe for Mn impurities in noble metals, which show similar spectra to those of our $(\mathrm{Ga}, \mathrm{Mn}) \mathrm{As}$ materials; for more details on the interpretation of the XMCD spectra see Ref. 16, and citations therein.

The Mn $3 d$ moments obtained from XMCD for the two (Ga,Mn)As samples are summarized in Table I. In both cases, magnetic moments of around $4.5 \mu_{B}$ are obtained, consistent with the SQUID results. Moreover, the measured moment and the property that it is independent of Mn doping is in very good agreement with the calculated $d$-projected magnetic moment shown in the inset of Fig. 4 and with the cor-

TABLE I. Mn $3 d$ moments obtained from XMCD and decomposed into the spin and orbital contributions in annealed samples with nominal Mn doping 2.2 and $8.4 \%$.

\begin{tabular}{cccc}
\hline \hline $\begin{array}{c}x \\
(\%)\end{array}$ & $\begin{array}{c}m_{\mathrm{XMCD}}^{\text {spin }} \\
\left( \pm 0.3 \mu_{B}\right)\end{array}$ & $\begin{array}{c}m_{\mathrm{XMCD}}^{\text {orb }} \\
\left( \pm 0.03 \mu_{B}\right)\end{array}$ & $\begin{array}{c}m_{\mathrm{XMCD}}^{\text {spin }}+m_{\mathrm{XMCD}}^{\text {orb }} \\
\left( \pm 0.3 \mu_{B}\right)\end{array}$ \\
\hline 2.2 & 4.3 & 0.15 & 4.5 \\
8.4 & 4.3 & 0.16 & 4.5 \\
\hline \hline
\end{tabular}


responding ab initio calculations reported in Refs. 35 and 36. We note here that all these microscopic calculations account only for the spin angular momentum contribution to the local Mn $3 d$ moment since spin-orbit coupling effects were neglected.

\section{CONCLUSIONS}

We report on a combined theoretical and experimental analysis of the spontaneous magnetization in $(\mathrm{Ga}, \mathrm{Mn}) \mathrm{As}$ DMS ferromagnets. We find that the thermodynamic magnetization is dominated by a large local moment contribution of $5 \mu_{B}$ from nearly collinear substitutional Mn atoms. Evaluation of the smaller magnetization contribution from the valence band system that couples the local moments together involves a number of subtleties. In this paper we included hole-hole exchange interactions and we also accounted for spin-orbit coupling which means that no valence band orbital is completely spin polarized and which substantially changes the overall electronic structure. Quantum fluctuations of the band and local moment orientations also play a role because of the antiferromagnetic interaction between band and local moment spins. The end result of all these corrections is a magnetization per magnetically active $\mathrm{Mn}$ ion that is suppressed from $5 \mu_{B}$ by $\sim 5-10 \%$. Comparison with experimental data can be made reliably only after accounting for the formation of interstitial Mn complexes during the MBE growth, and for their subsequent removal by post-growth annealing. Once these corrections have been applied, we find, within the experimental error bars, agreement between theory and experiment. The interpretation of XMCD magnetization measurements, which capture only the $d$-electron contribution, requires a recognition of the hybridized $p-d$ character of both local moment and band-electron contributions to the magnetization. Comparison of these measurements with TBA/CPA calculations provides experimental support for the applicability of the kinetic-exchange model in (Ga,Mn)As ferromagnets. Finally, our combined theoretical and experimental work demonstrates that noncollinearity does not play a significant role in the magnetization of highquality metallic $(\mathrm{Ga}, \mathrm{Mn})$ As ferromagnets.

\section{ACKNOWLEDGMENTS}

We thank Julio Cesare, Peter Bencok, and Nick Brookes for their contributions to the XMCD experiment. This work was supported by the Grant Agency of the Czech Republic through Grant No. 202/05/0575, by the Academy of Sciences of the Czech Republic through Institutional Support No. AV0Z10100521, by the Ministry of Education of the Czech Republic Center for Fundamental Research LC510, by the EU FENIKS Project No. EC:G5RD-CT-2001-00535, by the UK EPSRC through Grant No. GR/S81407/01, by the Welch Foundation, and the U.S. Department of Energy under Grant No. DE-FG03-02ER45958.
${ }^{1}$ H. Ohno and F. Matsukura, Solid State Commun. 117, 179 (2001).

${ }^{2}$ S. J. Potashnik, K. C. Ku, R. Mahendiran, S. H. Chun, R. F. Wang, N. Samarth, and P. Schiffer, Phys. Rev. B 66, 012408 (2002).

${ }^{3}$ P. Mahadevan, A. Zunger, and D. D. Sarma, Phys. Rev. Lett. 93, 177201 (2004).

${ }^{4}$ J. Kudrnovský, I. Turek, V. Drchal, F. Máca, P. Weinberger, and P. Bruno, Phys. Rev. B 69, 115208 (2004).

${ }^{5}$ C. Timm and A. H. MacDonald, Phys. Rev. B 71, 155206 (2005).

${ }^{6}$ J. König, T. Jungwirth, and A. H. MacDonald, Phys. Rev. B 64, 184423 (2001)

${ }^{7}$ J. Schliemann and A. H. MacDonald, Phys. Rev. Lett. 88, 137201 (2002).

${ }^{8}$ J. Schliemann, Phys. Rev. B 67, 045202 (2003).

${ }^{9}$ L. Brey and G. Gómez-Santos, Phys. Rev. B 68, 115206 (2003).

${ }^{10}$ G. Zaránd and B. Jankó, Phys. Rev. Lett. 89, 047201 (2002).

${ }^{11}$ G. A. Fiete, G. Zaránd, B. Jankó, P. Redliński, and C. P. Moca, Phys. Rev. B 71, 115202 (2005).

${ }^{12}$ H. Tsunetsugu, M. Sigrist, and K. Ueda, Rev. Mod. Phys. 69, 809 (1997).

${ }^{13}$ T. Jungwirth et al., Phys. Rev. B 72, 165204 (2005).

${ }^{14}$ T. Dietl, A. Haury, and Y. M. d'Aubigne, Phys. Rev. B 55, R3347 (1997).

${ }^{15}$ C. Zhou, M. P. Kennett, X. Wan, M. Berciu, and R. N. Bhatt, Phys. Rev. B 69, 144419 (2004).

${ }^{16}$ K. W. Edmonds, N. R. S. Farley, T. K. Johal, G. van der Laan, R. P. Campion, B. L. Gallagher, and C. T. Foxon, Phys. Rev. B 71,
064418 (2005).

${ }^{17}$ J. R. Schrieffer and P. A. Wolff, Phys. Rev. 149, 491 (1966).

${ }^{18}$ J. C. Slater and G. F. Koster, Phys. Rev. 94, 1498 (1954).

${ }^{19} \mathrm{~W}$. Harrison, Electronic Structure and the Properties of Solid (Freeman, San Francisco, 1980).

${ }^{20}$ D. N. Talwar and C. S. Ting, Phys. Rev. B 25, 2660 (1982).

${ }^{21}$ J. Mašek, B. Velický, and V. Janiš, J. Phys. C 20, 59 (1987).

${ }^{22}$ J. Mašek, Solid State Commun. 78, 351 (1991).

${ }^{23}$ J. Blinowski and P. Kacman, Phys. Rev. B 67, 121204(R) (2003).

${ }^{24}$ T. Jungwirth, J. Mašek, J. Sinova, and A. H. MacDonald, Phys. Rev. B 68, 161202(R) (2003).

${ }^{25}$ M. Abolfath, T. Jungwirth, J. Brum, and A. MacDonald, Phys. Rev. B 63, 054418 (2001).

${ }^{26}$ T. Dietl, H. Ohno, and F. Matsukura, Phys. Rev. B 63, 195205 (2001).

${ }^{27}$ J. G. Braden, J. S. Parker, P. Xiong, S. H. Chun, and N. Samarth, Phys. Rev. Lett. 91, 056602 (2003).

${ }^{28}$ A. K. Bhattacharjee, G. Fishman, and B. Coqblin, Physica B \& C 117-118, 449 (1983).

${ }^{29}$ J. Okabayashi, A. Kimura, O. Rader, T. Mizokawa, A. Fujimori, T. Hayashi, and M. Tanaka, Phys. Rev. B 58, R4211 (1998).

${ }^{30}$ J. Okabayashi, A. Kimura, O. Rader, T. Mizokawa, A. Fujimori, T. Hayashi, and M. Tanaka, Phys. Rev. B 64, 125304 (2001).

${ }^{31}$ A. Kaminski and S. Das Sarma, Phys. Rev. B 68, 235210 (2003).

${ }^{32}$ S. Das Sarma, E. H. Hwang, and A. Kaminski, Phys. Rev. B 67, 155201 (2003).

${ }^{33}$ M. Scarpulla, B. Cardozo, W. H. Oo, M. McCluskey, and O. Dubon (unpublished). 
${ }^{34}$ B. Velický, S. Kirkpatrick, and H. Ehrenreich, Phys. Rev. 175, 747 (1968).

${ }^{35}$ M. Wierzbowska, D. Sanchez-Portal, and S. Sanvito, Phys. Rev. B 70, 235209 (2004).

${ }^{36}$ T. Schulthess, W. M. Temmerman, Z. Szotek, W. Butler, and G. Stocks, Nat. Mater. 4, 838 (2005).

${ }^{37}$ T. Omiya, F. Matsukura, T. Dietl, Y. Ohno, T. Sakon, M. Motokawa, and H. Ohno, Physica E (Amsterdam) 7, 976 (2000).

${ }^{38}$ I. Vurgaftman, J. Meyer, and L. Ram-Mohan, J. Appl. Phys. 89, 5815 (2001).

${ }^{39}$ T. Dietl, H. Ohno, F. Matsukura, J. Cibert, and D. Ferrand, Science 287, 1019 (2000).

${ }^{40}$ R. Campion, K. Edmonds, L. Zhao, K. Wang, C. Foxon, B. Gallagher, and C. Staddon, J. Cryst. Growth 247, 42 (2003).

${ }^{41}$ C. T. Foxon, R. P. Campion, K. W. Edmonds, L. Zhao, K. Wang, N. R. S. Farley, C. R. Staddon, and B. L. Gallagher, J. Mater.
Sci. 15, 727 (2004).

${ }^{42}$ L. X. Zhao et al., Semicond. Sci. Technol. 20, 369 (2005).

${ }^{43}$ K. M. Yu, W. Walukiewicz, T. Wojtowicz, I. Kuryliszyn, X. Liu, Y. Sasaki, and J. K. Furdyna, Phys. Rev. B 65, 201303(R) (2002).

${ }^{44}$ K. Edmonds et al., Phys. Rev. Lett. 92, 037201 (2004).

${ }^{45}$ K. W. Edmonds, R. P. Campion, K.-Y. Wang, A. C. Neumann, B. L. Gallagher, C. T. Foxon, and P. C. Main, J. Appl. Phys. 93, 6787 (2003).

${ }^{46}$ K. W. Edmonds, N. R. S. Farley, R. P. Campion, C. T. Foxon, B. L. Gallagher, T. K. Johal, G. van der Laan, M. MacKenzie, J. N. Chapman, and E. Arenholz, Appl. Phys. Lett. 84, 4065 (2004).

${ }^{47}$ K. Edmonds, G. van der Laan, A. Freeman, N. Farley, T. Johal, R. Campion, C. Foxon, B. Gallagher, and E. Arenholz (unpublished).

${ }^{48}$ P. Carra, B. T. Thole, M. Altarelli, and X. Wang, Phys. Rev. Lett. 70, 694 (1993). 